\title{
Studies in the absorption and metabolism of vitamin $D$ after gastric surgery
}

\author{
GILBERT R. THOMPSON \\ Lecturer in Medicine, Royal Postgraduate Medical School, London, W.12
}

\section{Metabolism of vitamin $D$}

Vitamin $D$ is taken up from the intestinal lumen by the mucosa of the upper and midjejunum and is transported into lymph as the unaltered sterol (Schacter, Finkelstein \& Kowarski, 1964). A proportion of the absorbed vitamin $\mathbf{D}$ is metabolized within the intestine to an active metabolite (Norman, Lund \& de Luca, 1964). It is known that vitamin $D$ promotes the active transport of calcium across the intestinal mucosa (Schacter \& Rosen, 1959) and it has also been recently shown that vitamin $D$ initiates the formation of a calcium-binding protein at that site (Wasserman \& Taylor, 1966). One of the chief results of vitamin $D$ deficiency is a failure to properly mineralize osteoid tissue in bone and it is this aspect of vitamin D metabolism that $I$ shall mainly consider in this paper.

Previous studies on post-gastrectomy bone disease

During 1966 my colleagues and I at Hammersmith Hospital published the results of our investigations in a group of post-gastrectomy patients with a raised serum alkaline phosphatase (Thompson et al., 1966b). We found that bone biopsies from patients with a raised serum alkaline phosphatase (SAP) could be divided into two groups: (1) overt osteomalacia, and (2) apparently normal with no obvious increase in osteoid thickness. We next studied the plasma vitamin D-like activity in these two groups and found that all the osteomalacics and several of the patients with apparently normal bone biopsies had no detectable vitamin D-like activity. In order to assess the significance of these findings we gave an intravenous dose of vitamin $D_{3}$ to one of the patients with a raised SAP and low plasma vitamin D-like activity. Although this patient had an apparently normal bonebiopsy there was an obvious biochemical response to vitamin $D$ with an immediate raise in the serum phosphate and serum calcium and a subsequent fall in the SAP to normal. At this stage we concluded that this type of patient probably had a significant degree of vitamin D deficiency without any gross histological lesion of bone, and we called this situation sub-clinical osteomalacia.

Collaborative studies with Centre du Metabolisme Phosphocalcique, Paris

However, it seemed possible that our histological criteria were inadequate and so we, therefore, sent some of our biopsies to Dr Bordier, Dr Matrajt and Dr Hioco in Paris, where everything, including the bone histology is so elegant. They discovered some very interesting preliminary findings. The biopsies were taken, processed and stained with Solochrome Cyanine as described by Matrajt, Bordier \& Hioco (1966). This technique applied to normal bone clearly delineates calcified bone, osteoid and a wellmarked calcification front (see Fig. 1). In

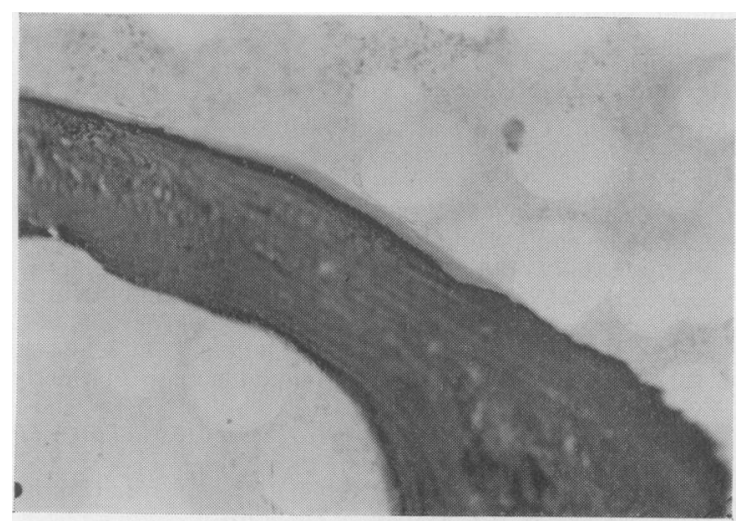

FIG. 1. Undecalcified section of normal bone stained with Solochrome Cyanine, showing a well-marked calcification front at the interface between calcified bone and osteoid.

osteomalacia the osteoid is present in excess and the calcification front is absent (Fig. 2), although it re-appears after treatment with vitamin D (Matrajt, Bordier \& Hioco, 1967). The really interesting findings concerned a patient with subclinical osteomalacia. The Paris workers showed 
that in this patient's bone-biopsy there was a virtual absence of the calcification front which, however, returned to normal after vitamin D therapy.

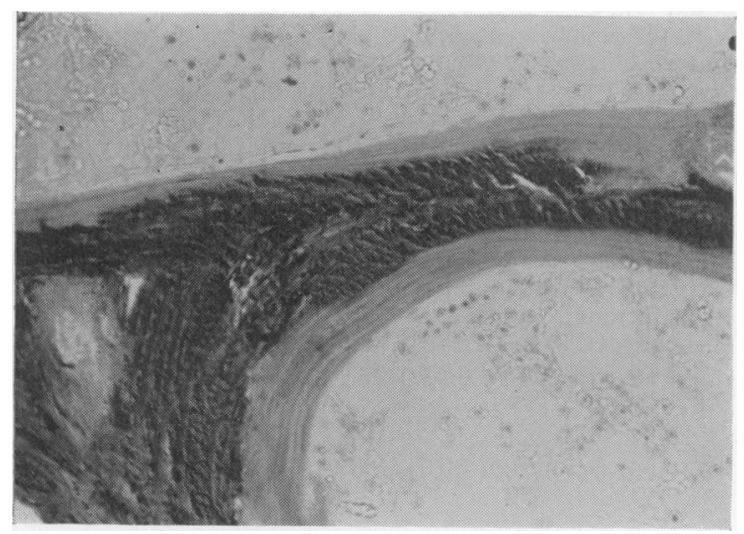

Fig. 2. Undecalcified section of bone from a case of osteomalacia, showing the increase in osteoid and absence of calcification front.

At this stage I departed to work in the U.S.A. but my colleague Dr Hepner continued the study with Dr Bordier and his colleagues. He has been taking biopsies both before and 8 days after an intravenous dose of vitamin $D_{3}$ from postgastrectomy subjects who agreed to co-operate in this study. The biopsies were sent to Paris and assessed by several criteria, including measurements of the proportion of bone surface covered with osteoid and the percentage of osteoid surfaces having a calcification front. The results of this study are reported in detail elsewhere (Bordier et al., 1968), but the main points are reviewed briefly below.

Osteoid surface. The osteoid surface area was linearly correlated with the SAP, and was increased in those post-gastrectomy patients who had a raised SAP. Each patient received an intravenous dose of $1 \mathrm{mg}$ of vitamin $D_{3} 8$ days before the second biopsy, but there was no change in the osteoid surface area.

Calcification front. The percentage of osteoid surfaces having a calcification front was linearly and inversely correlated with the SAP, and was decreased in patients with a raised SAP. Administration of intravenous vitamin $D$ caused a significant increase in the proportion of calcification fronts, both in patients with a raised SAP and in those with a normal SAP.
Relation of bone histology to serum alkaline phosphatase

It has been shown by Morgan et al. (1965) that a significant proportion of post-gastrectomy patients have a raised SAP. These authors also found that some of their patients had an excessive coverage of bone surface with osteoid. Our findings indicate that there is a positive correlation between the SAP and the percentage of bone-surface covered with osteoid and that there is a negative correlation between the SAP and the percentage of osteoid surface having a calcification front. Since the second abnormality is rapidly reversible with vitamin $D$, it seems likely that the decreased proportion of calcification fronts was due to vitamin $D$ deficiency in these patients. What we do not know is whether the increase in osteoid surface-area eventually returns to normal after vitamin $D$ nor whether it progresses to osteomalacia if left untreated.

\section{Studies of vitamin D absorption}

I should now like to briefly consider the subject of vitamin D absorption. Some time ago we carried out studies on the absorption of tritiumlabelled vitamin $D_{3}$ in patients with postgastrectomy osteomalacia (Thompson, Lewis \& Booth, 1966a). The 1-mg dose of vitamin D was given after a meal and was washed down with a glass of milk. The results showed that the osteomalacic subjects absorbed vitamin D only slightly less well than the controls. It seemed unlikely that this mild degree of malabsorption could have been solely responsible for these patient's osteomalacia, which raised the following question. Was the test valid and was the osteomalacia therefore mainly due to an inadequate intake of vitamin $\mathrm{D}$, or did the test fail to reflect the true extent of malabsorption of dietary vitamin D? It was difficult to know which of these alternatives provided the real answer so we temporarily deserted the human subject and turned to the rat.

During the past year I have been collaborating with Dr Ockner in Dr Isselbacher's laboratory at the Massachusetts General Hospital, Boston, in a study of the effect of dietary fat on sterol absorption in lymph-fistula rats. The labelled vitamin D was infused via an intraduodenal tube, dissolved in a mixed micellar solution of fatty acid, monoglyceride and bile salt. The results showed a linear correlation between the amount of radioactive vitamin $D$ and triglyceride appearing in lymph. In view of these results it seems possible that in man the absorption of vitamin $D$ may be dependent on the amount of dietary fat absorbed, and that a low fat intake might there- 
fore decrease vitamin $\mathrm{D}$ absorption. It would certainly seem worthwhile testing out this hypothesis by measuring vitamin $\mathrm{D}$ absorption in both control subjects and post-gastrectomy patients at varying levels of fat intake.

\section{References}

Bordier, P., Matrajt, H., Hioco, D., Hepner, G.W., Thompson, G.R. \& Booth, C.C. (1968) Subclinical vitamin D deficiency following gastric surgery. Lancet, $\mathbf{i}, 437$.

Matrajt, H. Bordier, H. \& Hioco, D. (1966) Solochrome cyanine $\mathbf{R}$ as an indicator dye of bone morphology. Stain Technol. 41, 97.

Matrajt, H., Bordier, P.H. \& Hioco, D. (1967) Mesures histologiques semi-quantitatives dans 17 observations d'osteomalacies nutritionnelles et renales. Influence de la Vitamine D (Ed. by D. J. Hioco), p. 101. L'Osteomalacie, Symposium organised by the Centre du Metabolisme Phospho Calcique. Masson, Paris.
Morgan, D.B., Paterson, C.R., Woods, C.G., Pulvertaft, C.N. \& Fourman, P. (1965) Search for osteomalaciai n 1228 patients after gastrectomy and other operations on the stomach. Lancet, ii, 1085.

Norman, A. W., Lund, J. \& De LuCA, H.F. (1964) Biologically active forms of vitamin $D_{3}$ in kidney and intestine. Arch. Biochem. Biophys. 108, 12.

SCHACTER, D. \& RoSEN, S.M. (1959) Active transport of Ca $\mathrm{Ca}^{45}$ by the small intestine and its dependence on vitamin $D$. Amer. J. Physiol. 196, 357.

Schacter, D., Finkelstein, J.O. \& Kowarski, S. (1964) Metabolism of vitamin D. I Preparation of radioactive vitamin $\mathrm{D}$ and its intestinal absorption in the rat. $J$. clin. Invest. 43, 787.

Thompson, G.R., Lewis, B. \& Booth, C.C. (1966a) Vitamin D absorption after partial gastrectomy. Lancet, i, 457.

Thompson, G.R., Neale, G., Watts, J.M. \& Booth, C.C. (1966b) Detection of vitamin D deficiency after partial gastrectomy. Lancet, ii, 623.

WAsSerman, R.H. \& TAYloR, A.N. (1966) Vitamin Dinduced calcium-binding protein in chick intestinal mucosa. Science, 152, 791. 